\title{
Smoothed Particles Hydrodynamics - the implementations of the incompressibility
}

\author{
(INDEX: 141-/2010 Copernican Letters ${ }^{\circledR}$ Vol 1) \\ Kamil Szewc \\ Institute of Fluid-flow Machinery, Polish Academy of Sciences, Gdańsk \\ ESSTIN, Université Henri Poincaré, Nancy, France \\ kamil.szewc@gmail.com
}

\begin{abstract}
The Smoothed Particle Hydrodynamics (SPH) is a fully Lagrangian, grid-free technique for fluid-flow simulations. An important and open issue in the SPH is a proper implementation of the incompressibility constraint. Generally there are two ways to deal with this problem. The first Weakly Compressible SPH (WCSPH) is the most widespread technique. It involves governing equations closed by a suitably-chosen equation of state. The second, Truly Incompressible SPH (ISPH), utilizes Projection Method - the technique known from the grid methods. The purpose of the present study is quantitative comparison of both techniques.
\end{abstract}

\section{Introduction}

The Smoothed Particle Hydrodynamics $(\mathrm{SPH})$ is a fully Lagrangian, particle-based technique for fluid-flow computations. In the early stage it was developed by Monaghan to simulate astrophysical phenomena [1], but nowadays, the SPH is increasingly often used for flows with interfaces and common in geophysical and astrophysical applications. The main advantage over Eulerian techniques is no requirement of the numerical grid, therefore there appears a spark of hope that the SPH method performs more accurate for complex geometries or multi phase flows. An important open issue in the $\mathrm{SPH}$ is a proper implementation of incompressibility constraint. In the present study, two different implementations are considered. The first is Weakly Compressible $\mathrm{SPH}$ (WCSPH), which is the most common technique. It involves the standard set of governing equations closed by a suitablychosen, artificial equation of state. The second implementation is performed by a truly compressible formulation based on the Projection Method (sec. 4.2). In this approach Incompressible PredictorCorrector (IPC) scheme is utilized. The common field of the research in the SPH is proper implementation of boundaries. Due to the incompressibility all the instabilities generated on the boundary quickly spread over all domain. In the present study, among many implementations, the ghostparticle boundary technique is applied. This approach involves the use of fictitious external particles that are mirrored by the fluid particles in the interior. The main advantages of this method are simplicity and conformity with different phases of the fluid [3].

\section{SPH formulation}

The main idea behind $\mathrm{SPH}$ is to introduce kernel interpolants for flow quantities so that the fluid dynamics is represented by particle evolution equations. The SPH technique is composed of two approximations. 
The first is interpolation of the fields at a point. To construct it, we utilize an integral interpolant $A_{I}(\boldsymbol{r})$ of any field $A(\boldsymbol{r})$

$$
A_{I}(\boldsymbol{r})=\int_{\Omega} A\left(\boldsymbol{r}^{\prime}\right) W\left(\boldsymbol{r}-\boldsymbol{r}^{\prime}, h\right) d \boldsymbol{r}^{\prime}
$$

where, the integration is over all the domain. $W$ is a weighting function (kernel) with a $h$, parameter (smoothing length) that can be treated as linear dimension of kernel. Generally, the kernel should posses the symmetrical form

$$
W(\boldsymbol{r}, h)=W(-\boldsymbol{r}, h)
$$

and enjoy following properties:

$$
\lim _{h \rightarrow 0} W(\boldsymbol{r}, h)=\delta(\boldsymbol{r})
$$

where $\delta(r)$ is the Dirac delta and should be normalized so that

$$
\int_{\Omega} W(\boldsymbol{r}, h) d r=1
$$

The additional condition is $W$ in $C^{n}$ where $n>0$ and at least as many times differentable as the field $A$ is. There are numerous possibilities to choose the kernel. Due to the computational effort and proper implementation of boundaries (sec. 5), we

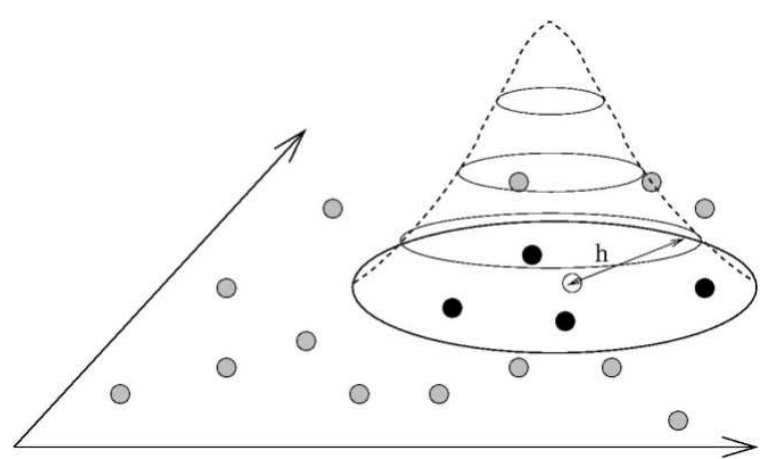

Figure 1. Set of neighboring particles. Due to the range of finite support (compact) kernel, only black particles interact with the white one.

decided to utilize the compact, quintic form (two-dimensional flows)

$$
W(\boldsymbol{r}, h)=\frac{7}{4 \pi h^{2}}\left(1-\frac{q}{2}\right)^{4}(2 q+1)
$$

where:

$$
q=\frac{|r|}{h}
$$

The second approximation of the $\mathrm{SPH}$ technique is discretization of space. It is done through dividing the domain into a fine-grained representation (particles). Each particle carries the properties of the field. The integral interpolant (2.1) becomes the summation interpolant

$$
A_{S}(\boldsymbol{r})=\sum_{a} A\left(\boldsymbol{r}_{a}\right) W\left(\boldsymbol{r}-\boldsymbol{r}_{a}, h\right) \Omega_{\mathrm{a}},
$$

where $\mathbf{r}_{a}$ and $\Omega_{a}$ denote the position and volume of the particle $a$. The SPH task consists in computing foregoing interpolant at each particle (Fig. 1), so that Eq. (2.7) may be rewritten into the common form 


$$
A_{a}=\sum_{b} A_{b} W_{a b}(h) \Omega_{\mathrm{b}}
$$

where:

$$
A_{a}=A\left(\boldsymbol{r}_{a}\right)
$$

and

$$
W_{a b}(h)=W\left(\boldsymbol{r}_{a}-\boldsymbol{r}_{b}, h\right)
$$

An additional quality of the SPH reveals with differentiation of the fields. In accordance with (2.1), the gradient of $A(r)$ assumes the form

$$
\nabla A(\boldsymbol{r})=\int_{\Omega} \nabla A\left(\boldsymbol{r}^{\prime}\right) W\left(\boldsymbol{r}-\boldsymbol{r}^{\prime}\right) d \boldsymbol{r}^{\prime} \cdot(2.11)
$$

Taking the advantage of the integration by parts rule and utilizing kernel symmetry, we can transform foregoing equation into

$$
\begin{aligned}
& \nabla A(\boldsymbol{r})=\left.A\left(\boldsymbol{r}^{\prime}\right) W\left(\boldsymbol{r}-\boldsymbol{r}^{\prime}\right)\right|_{\partial \Omega} \\
& +\int_{\Omega} A\left(\boldsymbol{r}^{\prime}\right) \nabla W\left(\boldsymbol{r}-\boldsymbol{r}^{\prime}\right) d \boldsymbol{r}^{\prime} .
\end{aligned}
$$

Generally, the first term does not necessarily vanish for finite systems. The common way is to remove this term and deal with the boundaries explicitly. The $\mathrm{SPH}$ form (discretization) of (2.11) brings the common rule

$$
\nabla A_{a}=\sum_{b} A_{b} \nabla W_{a b}(h) \Omega_{\mathrm{b}}
$$

Since the nabla operator acts only on the kernel, the gradient of the field is dependent only on the values of the fields at particles, not gradients.

The way of obtaining higher derivatives is straightforward. The Laplacian operator which acts on field $A(r)$ has the form

$$
\Delta A_{a}=\sum_{b} \sum_{c} A_{c} \nabla W_{a b}(h) \nabla W_{b c}(h) \Omega_{\mathrm{b}} \Omega_{\mathrm{c}} .
$$

However, due to the accuracy and efficiency, commonly utilized form is built as a combination of the finite difference approach and the SPH [4][5] (Sec. 3).

\section{Governing equations}

The full set of governing equations for incompressible viscous flow is composed of the Navier-Stokes equation

$$
\frac{d \boldsymbol{u}}{d t}=-\frac{1}{\varrho} \nabla p+\nabla(v \nabla \boldsymbol{u})+\boldsymbol{f}
$$

where $\rho$ is the density, $\boldsymbol{u}$ the velocity vector, $t$ the time, $p$ the pressure, $v$ the kinematic viscosity and $f$ an external force, and the divergence-free constraint

$$
\nabla \boldsymbol{u}=0 .
$$

Equation (3.1) arises from applying the Newton's Second Law to fluid motion with 
assumption that the fluid stress is the sum of viscous and pressure terms. The continuity equation (the conservation of mass) has the form

$$
\frac{d \varrho}{d t}=-\varrho \nabla \boldsymbol{u} .
$$

In the case of incompressible flow, density $\varrho$ is constant, therefore Eq. (3.3) arises to the form (3.2).

The whole set of governing equations should be expressed in the $\mathrm{SPH}$ approach. Utilizing relation (2.12), divergence of velocity takes the SPH form

$$
\nabla \boldsymbol{u}_{a}=\sum_{b} \boldsymbol{u}_{b} \nabla_{\mathrm{a}} W_{a b}(h) \Omega_{\mathrm{b}}
$$

Therefore, the continuity equation (3.3) has the form

$$
\frac{d \varrho_{a}}{d t}=-\varrho_{a} \sum_{b} \boldsymbol{u}_{b} \nabla_{\mathrm{a}} W_{a b}(h) \frac{m_{b}}{\varrho_{b}},
$$

where

$$
\frac{m_{b}}{\varrho_{b}}=\Omega_{\mathrm{b}}
$$

It is important to note that various ways to express divergence exist, for example, using the identity

$$
\nabla \boldsymbol{u} \equiv \frac{1}{\varrho}(\nabla(\varrho \boldsymbol{u})-\boldsymbol{u} \nabla \varrho)
$$

Combining (3.3) with (3.7) leads to the different SPH form

$$
\frac{d \varrho_{a}}{d t}=\sum_{b} m_{b} \boldsymbol{u}_{a b} \nabla_{\mathrm{a}} W_{a b}(h),
$$

where $\boldsymbol{u}_{a b}=\boldsymbol{u}_{a}-\boldsymbol{u}_{b}$. The advantage of the above form over (3.5) is the symmetry with swapping particles a and b. Therefore, in practice, it is more accurate to use (3.8) [8]. However, there exists an alternative formulation. The fluid density can be computed directly from the SPH formula (2.8)

$$
\varrho_{a}=\sum_{b} \varrho_{b} W_{a b}(h) \Omega_{\mathrm{b}}=\sum_{b} m_{b} W_{a b}(h)
$$

A practical disadvantage of this approach is that $\varrho$ must be evaluated by summing over the particles before other quantities [9]. Therefore it increases the computational effort.

The right hand side of the NavierStokes equation (3.1) contains three elements: pressure, viscous and external force term. In the SPH technique the pressure term is responsible for ensuring the incompressibility constraint (Sec. 4). Utilizing (2.12) it takes the form

$$
\frac{1}{\varrho_{a}} \nabla_{\mathrm{a}} p_{a}=\frac{1}{\varrho_{a}} \sum_{b} p_{b} \nabla_{\mathrm{a}} W_{a b}(h) \frac{m_{b}}{\varrho_{b}} .
$$


Similarly to the continuity equation (3.5) it is more accurate to use another form. Utilizing the identity

$$
\nabla p \equiv \varrho\left(\nabla\left(\frac{p}{\varrho}\right)+\frac{p}{\varrho^{2}} \nabla \varrho\right)
$$

the gradient of pressure can be expressed as

$$
\frac{1}{\varrho_{a}} \nabla_{a} p_{a}=\frac{1}{\varrho_{a}} \sum_{b} m_{b}\left(\frac{p_{a}}{\varrho_{a}^{2}}+\frac{p_{b}}{\varrho_{b}^{2}}\right) \nabla_{a} W_{a b}(h) .
$$

This form ensures angular momentum conservation [1].

The viscous term, as mentioned in Sec. 2, is built as a combination of the finite difference approach and the SPH. For present work, we utilize the form [5]

$$
\begin{gathered}
\nabla\left(v_{a} \nabla \boldsymbol{u}_{a}\right) \\
=8 \sum_{b} m_{b}\left(\frac{v_{a}+v_{b}}{\varrho_{a}+\varrho_{b}} \frac{\boldsymbol{u}_{a b} \boldsymbol{r}_{a b}}{r_{a b}^{2}+\eta^{2}}\right) \nabla_{\mathrm{a}} W_{a b}(h) .
\end{gathered}
$$

Since the SPH is fully Lagrangian approach, the particle equation of motion completes the system

$$
\frac{d \boldsymbol{r}_{a}}{d t}=\boldsymbol{u}_{a}
$$

\section{Incompressibility treatment}

Generally, there are two techniques to assure the incompressibility in the SPH.

\subsection{Weakly Compressible SPH}

The most common technique is WCSPH. It involves the set of governing equations closed by a suitably-chosen, artificial equation of state $p=p(\rho)$. Since fluid pressure is an explicit function of $\rho$, density gradient exerts an influence at particle motion. The commonly used equation of state has the form suggested by Batchelor [6]

$$
p=\frac{c^{2} \varrho_{0}}{\gamma}\left[\left(\frac{\varrho}{\varrho_{0}}\right)^{\gamma}-1\right]
$$

where reference density $\rho_{0}$, numerical sound speed $c$ and parameter $\gamma$ are suitablychosen to reduce the density fluctuation down to $1 \%$. For present work we keep $\gamma=7$ and $c$ at the level minimum 10 times higher than the maximal fluid velocity. Since, assuring incompressibility constraint, the sound speed is high, the time step should be very small. This is the potential weakness of the WCSPH.

\subsection{Truly Incompressible SPH}

The newest promising technique is truly Incompressible SPH (ISPH). It is based on the Projection Method - common approach for numerically solving timedependent incompressible fluid-flow problems. In this technique the pressure needed to ensure incompressibility is found by projecting calculated velocity field onto 
the divergence-free space [4]. It is possible due to the Helmholtz decomposition which states: every vector field $A$, that is twice continuously differentable and vanishes faster than $1 / r$ at infinity, can be decomposed into gradient and curl as follows [7]

$$
\boldsymbol{A}=\nabla \phi+\nabla \times \boldsymbol{B}=\boldsymbol{A}_{\text {curl free }}+\boldsymbol{A}_{\text {div free, }}
$$

where $\phi$ and $\boldsymbol{B}$ are suitably chosen and

$$
\begin{aligned}
\nabla \boldsymbol{A}_{\text {div free }} & =\nabla(\nabla \times \mathrm{B})=0, \\
\nabla \times \boldsymbol{A}_{\text {curl free }} & =\nabla \times(\nabla \phi)=0 .
\end{aligned}
$$

In the ISPH, decomposition procedure begins with splitting the Navier-Stokes equation (3.1) into two parts. The first, socalled predictor step gives the fractional velocity $\boldsymbol{u}^{*}$

$$
\frac{\boldsymbol{u}^{*}-\boldsymbol{u}^{n}}{\delta t}=\nabla(v \nabla \boldsymbol{u})+\boldsymbol{f} .
$$

This equation excludes the influence of the pressure $p$ - we treat pressure as nonphysical quantity. Therefore in this approach all real, physical pressure forces are enclosed in the $f$ term. The second part of the procedure is the correction step

$$
\frac{\boldsymbol{u}^{n+1}-\boldsymbol{u}^{*}}{\delta t}=-\frac{1}{\varrho} \nabla p^{n+1} .
$$

It imposes the correction to $u^{*}$ for ensuring compliance with the divergence-free constraint. On the way to obtain an appropriate quantity $p^{n+1}$ we write the divergence of Eq. (4.5) (projection into divergence-free space)

$$
\nabla\left(\frac{\boldsymbol{u}^{n+1}-\boldsymbol{u}^{*}}{\delta t}\right)=\nabla\left(-\frac{1}{\varrho} \nabla p^{n+1}\right)
$$

Since we expect divergence-free velocity field, we have $\operatorname{div} \mathbf{u}^{\mathrm{n}+1}=0$. Therefore, the formula (4.6) leads to the Poisson equation

$$
\nabla\left(\frac{1}{\varrho} \nabla p^{n+1}\right)=\frac{\nabla \boldsymbol{u}^{*}}{\delta t}
$$

Now, the correction step (4.5) performed with $p^{n+1}$ obtained from above relation, gives the divergence-free velocity field at the next time step.

\subsubsection{The Particle Poisson Solver}

Since the SPH is a particle-based method, there exists specific treatment of the Poisson equation. Instead of solving Eq. (4.7) on a regular grid, we may approximate the Laplacian operator with the same form as a viscous term (3.13) in the Navier-Stokes equation (see Cummins and Rudman [2]). In this concept the Poisson equation is solved on irregular grid of Lagrangian points (particles). But since the authors of the paper [14] report that the Particle Poisson Solver (PPS) has the order of $1 / 2$ and the order decreases increasing the number of particles in the system, for further work, we decided to utilize the Poisson solver on a regular grid. 
a)

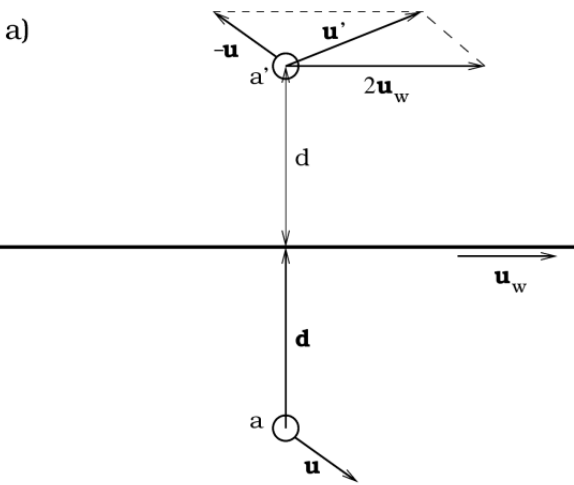

b)

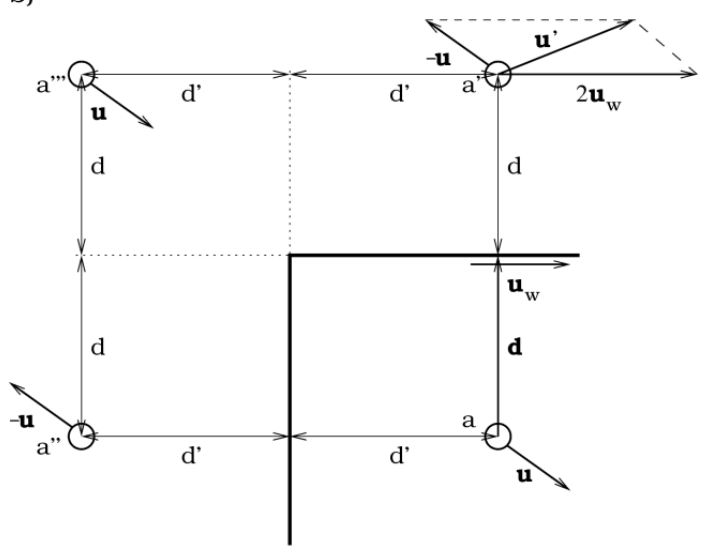

Figure 2 . The ghost-particle boundary scheme.

\section{Boundary condition}

The proper implementation of the boundaries is one of the most common subjects of the SPH research during recent years. Early stage applications of WCSPH involved high Reynolds number simulations with free-slip boundaries. It was performed using one layer of the boundary particles placed on the wall, which exert strong repulsive force to prevent from penetrating solid surfaces [10]. Since the number of interacting particles near the walls is decreased, the accuracy of numerical scheme degrades. Another treatment was proposed by Campbell [11]. The boundary condition was included already in Eq. (2.11) through the residual term. Today, generally, the most often used boundary condition utilizes dummy particles [12]. In this approach, virtual boundary particles are regularly distributed according to initial configuration of the particles and have zero velocity during whole simulations (no-slip condition). Other popular virtual-particle based boundary conditions utilize mirror particles. These particles in opposition to dummy particles, have suitably chosen nonzero velocity. Nowadays, there are two commonly used mirror-particle approaches. The first, developed by Morris [9], consists in the combination of dummy and mirror particles. The velocity of fluid particles is suitably projected on fixed boundary particles. The second approach so-called Multiple Boundary Tangent (MBT) method is similar to the previous but the way of projection particles' velocities is different.

Presented mirror-particle techniques, was developed to improve another, more natural approach - the ghost particle method. This technique is similar to the Classic Image Problem in electrostatics [7]. To any particle $a$ located at $\boldsymbol{r}_{a}$ near the straight infinite boundary, we introduce the image $a^{\prime}$ of this particle with the following properties:

$$
\begin{gathered}
\boldsymbol{r}_{a^{\prime}}=2 \boldsymbol{d}+\boldsymbol{r}_{a}, \\
\boldsymbol{u}_{a^{\prime}}=2 \boldsymbol{u}_{w}-\boldsymbol{u}_{a}, \\
m_{a^{\prime}}=m_{a}, \\
\varrho_{a^{\prime}}=\varrho_{a}, \\
p_{a^{\prime}}=p_{a},
\end{gathered}
$$

where $\boldsymbol{u}_{\mathrm{w}}$ is the velocity of the boundary and $\boldsymbol{d}$ is a vector between the particle and the nearest point at the wall. Described construction is presented in Fig. 2a. Since chosen kernel is compact, the boundary may be finite. The role of these particles is 
to assure high accuracy of the computation (to replenish the lack of particles near the boundaries) and to enforce the boundary condition for velocity (no-slip), density and pressure.

Another case that can be treated with ghost-particle boundary approach is an inner corner. The technique of constructing particles' images is presented in Fig. 2b. In this case we have to use three mirror particles with the following properties

$$
\begin{gathered}
\boldsymbol{r}_{a \prime}=2 \boldsymbol{d}+\boldsymbol{r}_{a} \\
\boldsymbol{r}_{a \prime \prime}=2 \boldsymbol{d}^{\prime}+\boldsymbol{r}_{a} \\
\boldsymbol{r}_{a^{\prime \prime \prime}}=2 \boldsymbol{d}+2 \boldsymbol{d}^{\prime}-\boldsymbol{r}_{a} \\
\boldsymbol{u}_{a \prime}=2 \boldsymbol{u}_{w}-\boldsymbol{u}_{a} \\
\boldsymbol{u}_{a \prime \prime}=-\boldsymbol{u}_{a} \\
\boldsymbol{u}_{a \prime \prime \prime}=\boldsymbol{u}_{a} \\
m_{a \prime}=m_{a \prime \prime}=m_{a \prime \prime \prime}=m_{a} \\
\varrho_{a \prime}=\varrho_{a \prime \prime}=\varrho_{a \prime \prime \prime}=\varrho_{a} \\
p_{a \prime}=p_{a \prime \prime}=p_{a \prime \prime \prime}=p_{a}
\end{gathered}
$$

It is important to note that the influence range of this corner is smaller than $2 \mathrm{~h}$. For larger distance from the corner, the boundary condition get back to the previous case (both in vertical and horizontal direction).

\section{Results}

In the present study, we describe two flows which are commonly utilized as numerical algorithms' tests: the Couette flow and the lid-driven cavity. In both of them, the

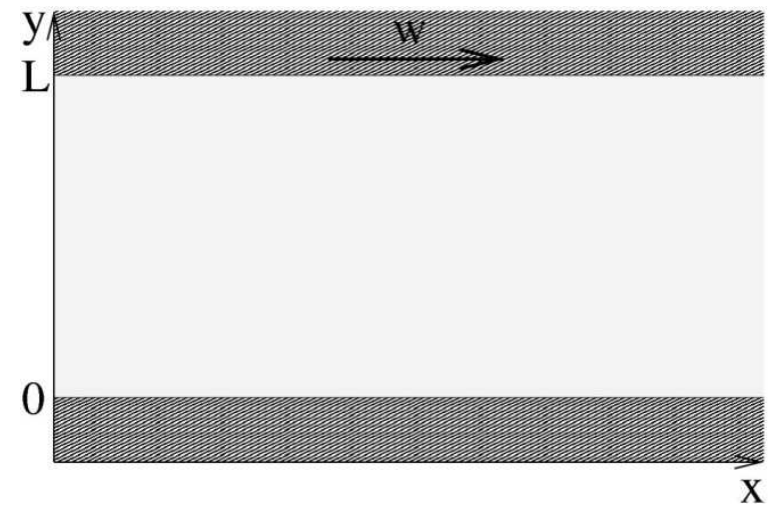

Figure 3. Scheme of the Couette flow.

domain contains 3600, 1600 and 400 particles placed homogeneously at the beginning of the simulations.

Limiting the time of computation, we obtain the density in the WCSPH with the formula (3.8). In the ISPH, the Poisson solver (4.7) is performed on the regular mesh with the number of nodes equal to the one quarter of particles' number. For the present work we utilize the five-point iterative solver.

As a quantitive measurement of incompressibility we compute the mean density $\langle\rho(t)>$ and the root mean square of density fluctuations RMS $(\rho(t))$ both performed on regular sets of nodes. The RMS coefficient is defined as

$$
R M S(\varrho(t))=\sqrt{\frac{1}{N} \sum_{i=1}^{N}\left(\varrho_{i}-\left\langle\varrho_{i}\right\rangle\right)^{2}},
$$

where $N$ denotes the number of nodes. For the present work, this number is equal to the number of particles. The higher values of $\operatorname{RMS}(\rho(t))$ coefficient stand for higher variations of density and, therefore, larger departures from the incompressibility. All the quantities (except the CPU time) are expressed in a non-dimensional form. 

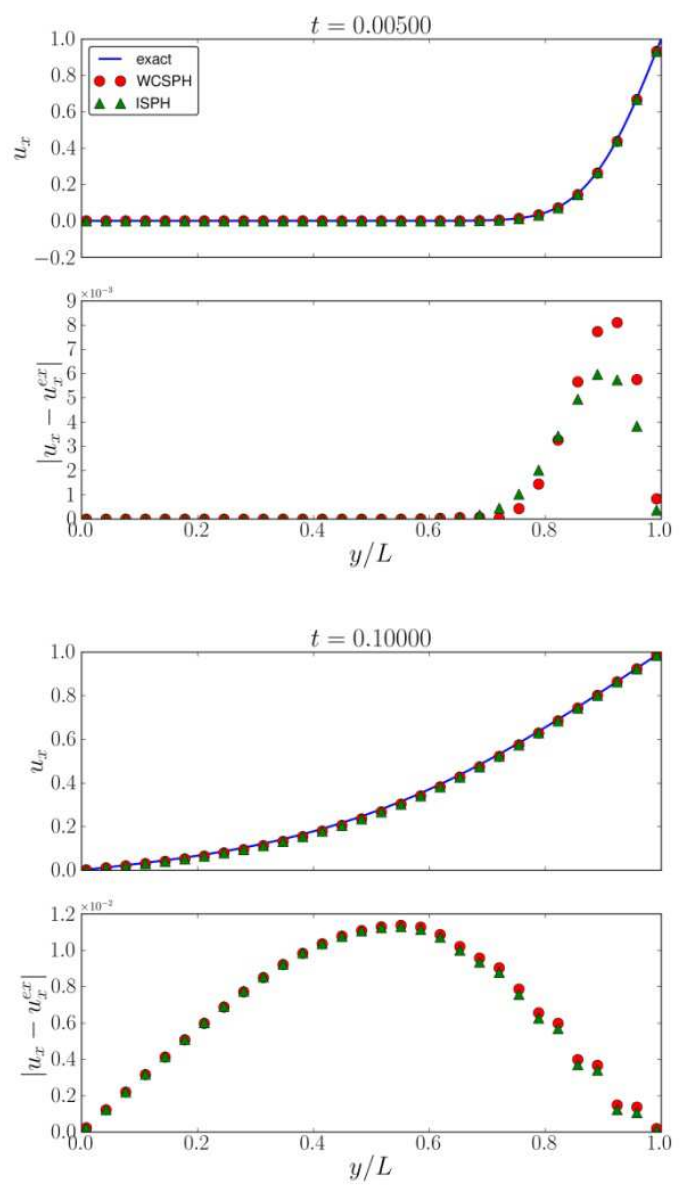
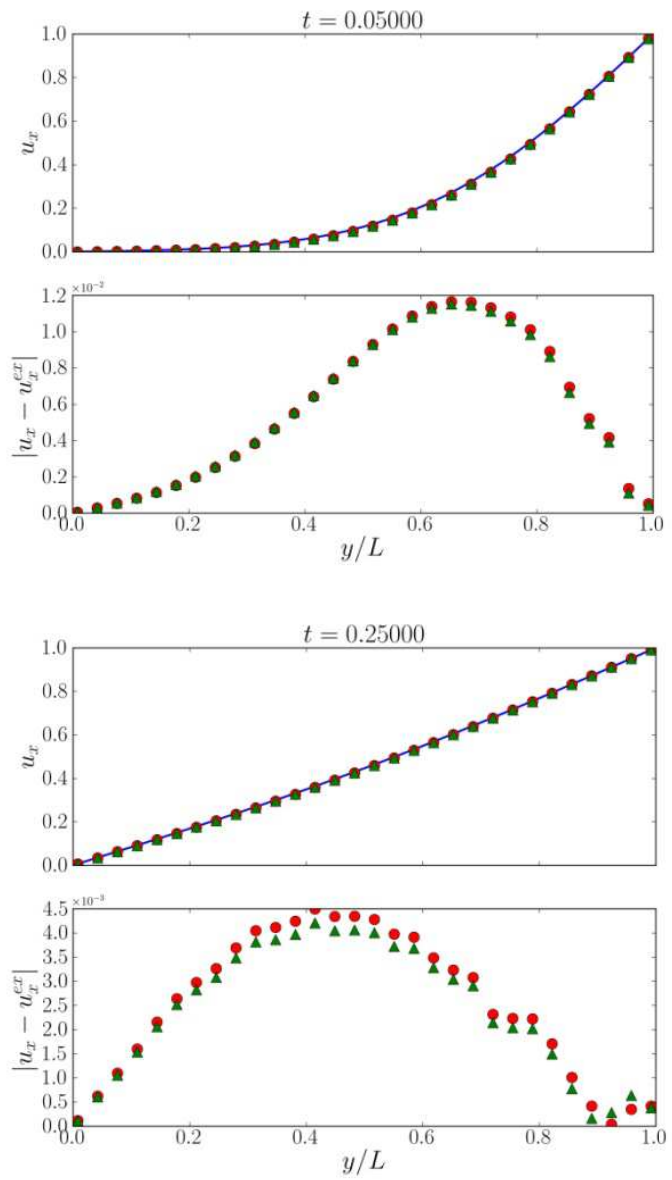

Figure 4. The velocity profiles and local deviation of Couette flow obtained with Weakly Compressible SPH (WCSPH) and Truly Incompressible SPH (ISPH) for $N=\mathbf{3 6 0 0}$ particles. Solid line presents the exact solution Eq. (6.6).

\subsection{Couette flow}

The Couette flow is classic, and simple, and due to the analytic solvability grateful for testing the numerical simulations. It involves viscous laminar flow between two parallel plates fixed at $y=0$ and $y=L$ where one of them moves with constant velocity $w$ in the $\mathrm{x}$ direction. Scheme of the Couette flow is presented in Fig. 3. The solution of the velocity field can be obtained by solving the Navier-Stokes equation (3.1). Since, due to the symmetry, the velocity field is not depended on the $x$ direction, the steady state solution reduces Eq. (3.1) into the form

$$
\Delta u_{x}^{\mathrm{ex}}(y)=0 .
$$

Integrating twice, we obtain

$$
u_{x}^{\mathrm{ex}}(y)=A y+B
$$

where $A$ and $B$ are constants that can be obtained using boundary conditions:

$$
\begin{aligned}
& u_{x}(0)=0, \\
& u_{x}(L)=w .
\end{aligned}
$$



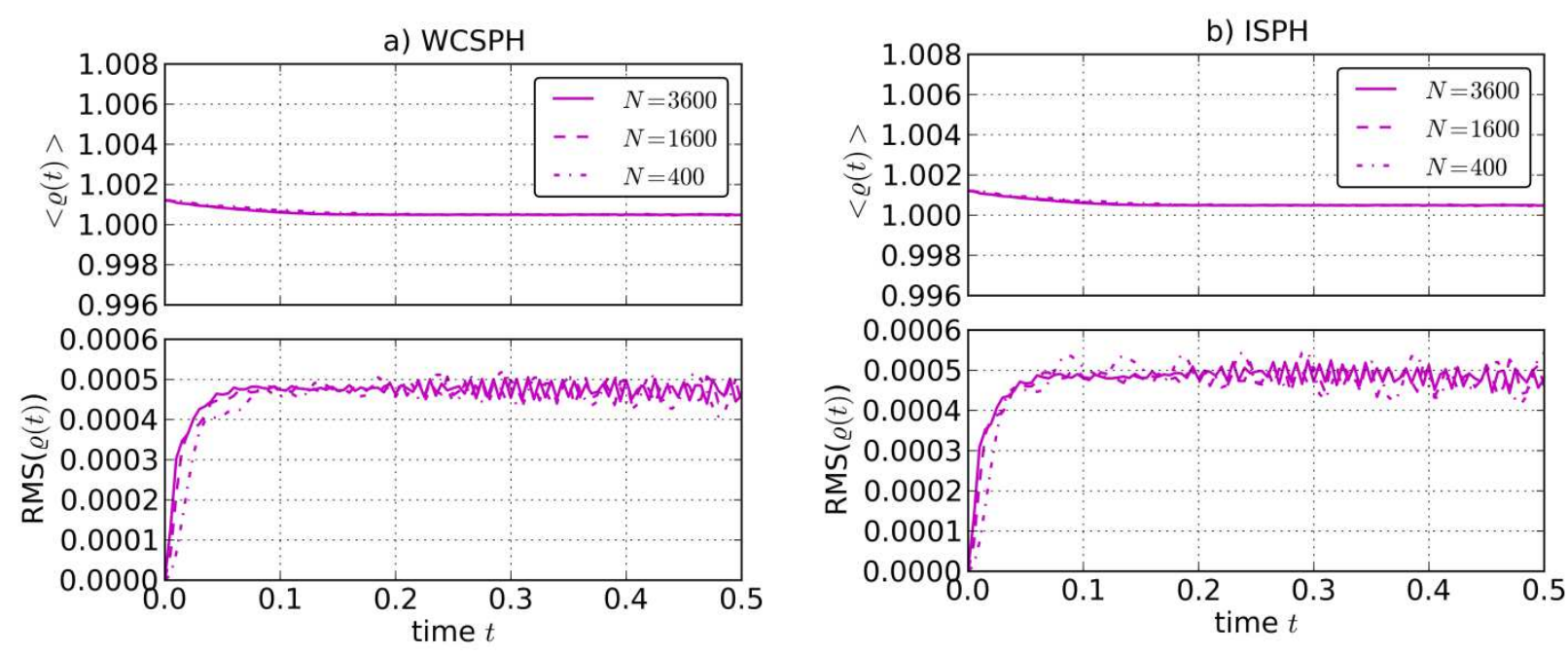

Figure 5. The density mean value and RMS coefficient obtained for the Couette flow (Re=1) performing: a) WCSPH and b) ISPH technique. Parameter $N$ denotes the number of particles in the domain.

Bringing into play above conditions, the steady-state solution of the Couette flow arises into the form

$$
u_{x}^{\mathrm{ex}}(y)=\frac{w}{L} y
$$

Time-dependent solution can be obtained utilizing a series formula. It takes following form

$$
\begin{aligned}
& u_{x}^{\mathrm{ex}}(y, t)=\frac{w}{L} y+\sum_{n=1}^{\infty} \frac{2 w}{n \pi}(-1)^{n} \times \\
& \times \sin \left(\frac{n \pi y}{L}\right) \exp \left(\frac{-v n^{2} \pi^{2}}{L^{2}} t\right) .
\end{aligned}
$$

$=\quad$ The Reynolds number that gives a measure of the ratio of inertial forces to viscous forces is defined as

$$
R e=\frac{v L}{v}
$$

where $v, L$ and $v$ are characteristic velocity, length and kinetic viscosity of considered flow. For the present test, the velocity of upper boundary was $w=1$, the kinetic viscosity $v=1$ and the distance between bottom and top boundary $\mathrm{L}=1$. Therefore, the Reynolds number is equal to 1.

The results of SPH simulations are presented in Fig. 4. To compare the accuracy of SPH implementations, we introduce the local deviation function, defined as

$$
\delta u_{x}\left(y_{i}^{n}\right)=\left|u_{x}\left(y_{i}^{n}\right)-u_{x}^{\mathrm{ex}}\left(y_{i}^{n}\right)\right|
$$

where $\mathrm{u}^{\mathrm{ex}}\left(\mathrm{yi}^{\mathrm{n}}\right)$ is the exact solution of the Couette flow (Eq. (6.6)) at position $y$, after $n$ timesteps. As we can see (fig. 4), the accuracy of velocity in the WCSPH as well as in the ISPH is at similar level. Since the Couette flow in our case is driven only by the upper boundary and the geometry is simple, the stream lines are parallel. Therefore, due to the lack of outer, real 


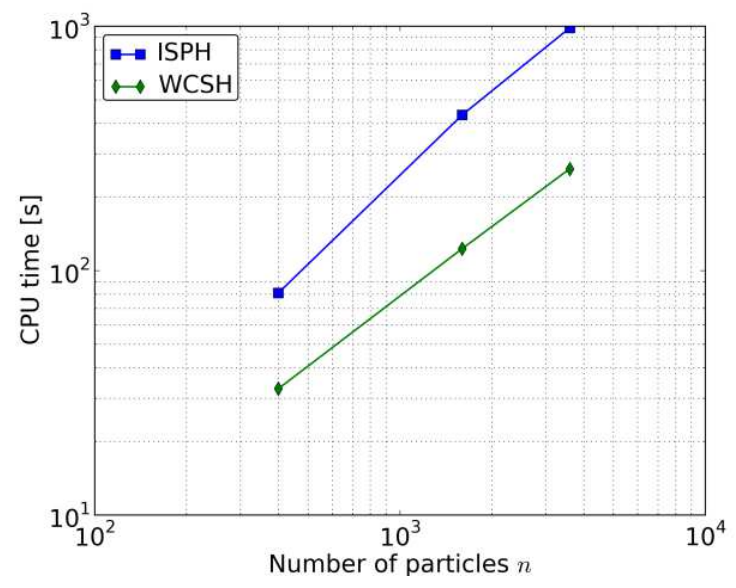

Figure 6 . The CPU times to obtain steady-state $(\mathrm{t}=0.25)$ solution of the Couette flow $(\operatorname{Re}=1)$.

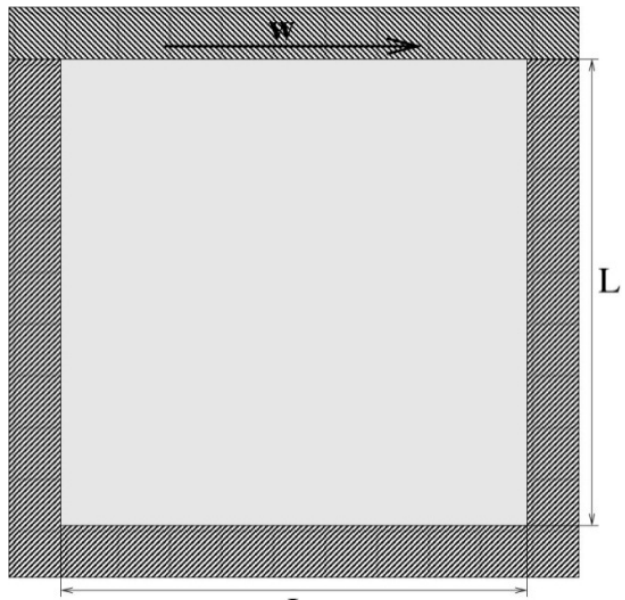

Figure 7. The scheme of the lid-driven cavity.

pressure forces, the pressure term in the Navier-Stokes equation does not provide significant influences. Due to this properties, the RMS of density (fig. 5), in both techniques, is at the similar, extremely small level. After $t=0.2$, when we obtains the steady-state solution, the RMS of density became stable around $0.7 \%$ of initial density. Even the varying number of particles doesn't radically change the RMS coefficient.

The comparison of computational times, presented in Fig. 6, suggests a greater efficiency of the WCSPH. But as was mentioned above, since there is no internal forces (Navier-Stokes pressure term) in the Couette flow, the equation of state (4.1) in the case of WCSPH and the correction step (4.5) with Poisson solver (4.7) in ISPH, are useless. Therefore, these parts of algorithm take up the CPU time only. Since the CPU time utilized by Eq. (4.1) is negligible in the comparison with solving Eq. (4.7), the WCSPH and ISPH computational time difference is the utilization of CPU time by the Poisson solver.

\subsection{Lid-driven cavity flow}

The lid-driven cavity is a very common test of numerical algorithms for viscous flows. It involves fluid-flow inside a square (LxL) box (presented on Fig. 7) where only one boundary moves with the velocity $w$. The geometry is very simple, however there is no analytical solution. For our tests we computed the lid-driven cavity at $R e=1000$. All the results obtained in the present work are compared to the results of numerical calculation on a fine grid performed on the Eulerian-flow solver by Ghia et al. [13]. Due to the sound speed constraint in the WCSPH technique, the necessary time step was $\delta \mathrm{t}=0.325 \cdot 10^{-4}$. For the Incompresible SPH $\delta \mathrm{t}=0.325 \cdot 10^{-2}$ is enough.

The steady-state velocity profiles for both methods are presented in Figs. 8 and 9 . As we can see, the profiles obtained via the ISPH and the WCSPH are similar. However, for $N=3600$ particles, ISPH technique are closer to the results obtained by Ghia et al., then performed via WCSPH. Another situation takes a place for $N=400$. For this case, the WCSPH seems to perform more accurately. 
Comparing the RMS of density coefficient (Fig. 10) it is easy to observe that the WCSPH solver deals much more accurately with density field than the ISPH. The RMS of density coefficient, obtained for ISPH solver, stabilizes after about $t=4$. Then, the RMS are equal: for $N=3600$ particles about $4.5 \%$, for $N=1600$ about $5.5 \%$ and for $N=400$ about 3\% (sic!) of initial density. The discrepancy between above values is caused by two factors: the number of particles and different number of nodes in Poisson solver's grid. The decreasing number of particles leads to higher errors of quantities but the decreasing number of nodes in Poisson solver smoothes out the density field. The value of RMS coefficient in the case of WCSPH is much under $1 \%$. The difference in accuracy of density field between discussed methods is caused by the disparity in the time steps. The time step in the WCSPH is 100 times smaller than the time step chosen to perform the ISPH.

Due to the above-mentioned disparity in the time steps, the CPU time of the liddriven cavity simulation computed by the ISPH method is about 20 times shorter than the same simulation performed with the WCSPH. The comparison of CPU times for both techniques is presented in Fig. 11.

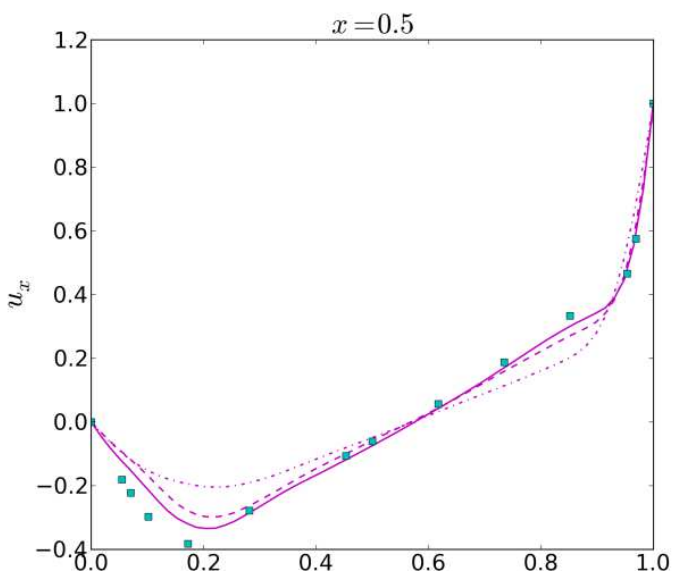

Figure 8. The WCSPH lid driven cavity velocity profiles at $t=55.0$ against Ghia et al. results. The results for different number
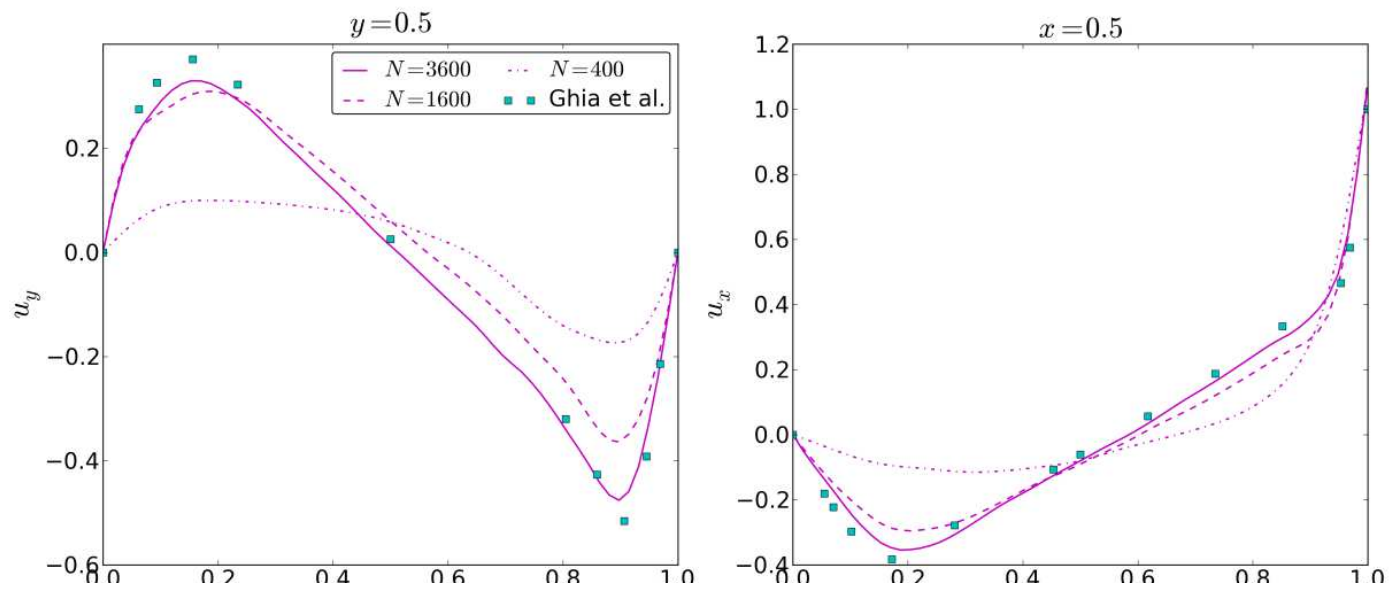

Figure 9. The ISPH lid-driven cavity velocity profiles at $t=55.0$ against Ghia et al. results. 


\section{Conclusions}

In this paper the Couette flow and the liddriven cavity flow have been simulated to compare the WCSPH and the ISPH techniques. In the considered lid-driven cavity test, accuracy of the velocity field is greater in the WCSPH than the ISPH in the case of small number of particles. When the number of particles increase, the accuracy of the ISPH technique increase faster than the WCSPH and for the number $N=3600$.

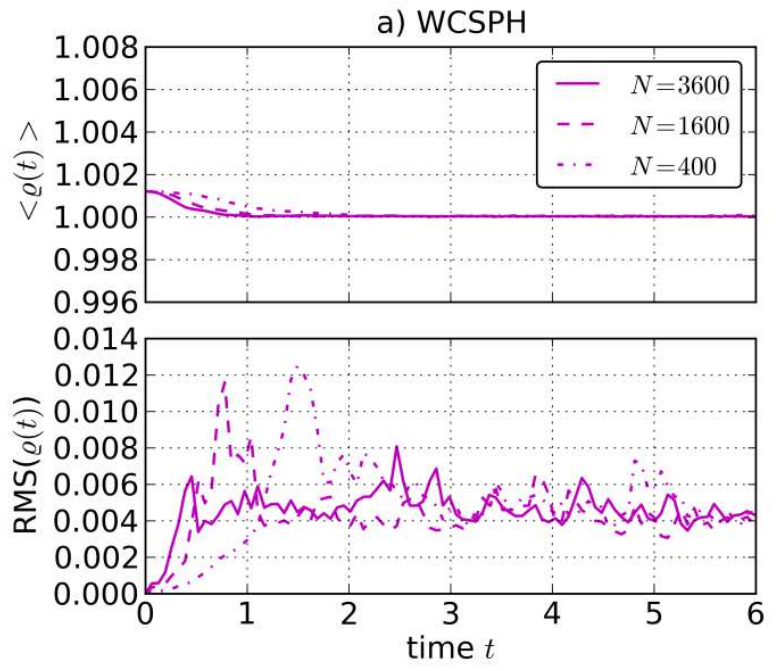

Further work may be undertaken to improve the ISPH technique. The utilization of faster Poisson solvers such as preconditioned Bi-CGSTAB is bound to get higher efficiency. Additional work is necessary to make quantitative comparison of the ISPH technique with the Poisson equation solved on grid and via PPS.

Qualitatively new approach of the ISPH was suggested by Pozorski and Wawreńczuk in paper [15]. Besides the

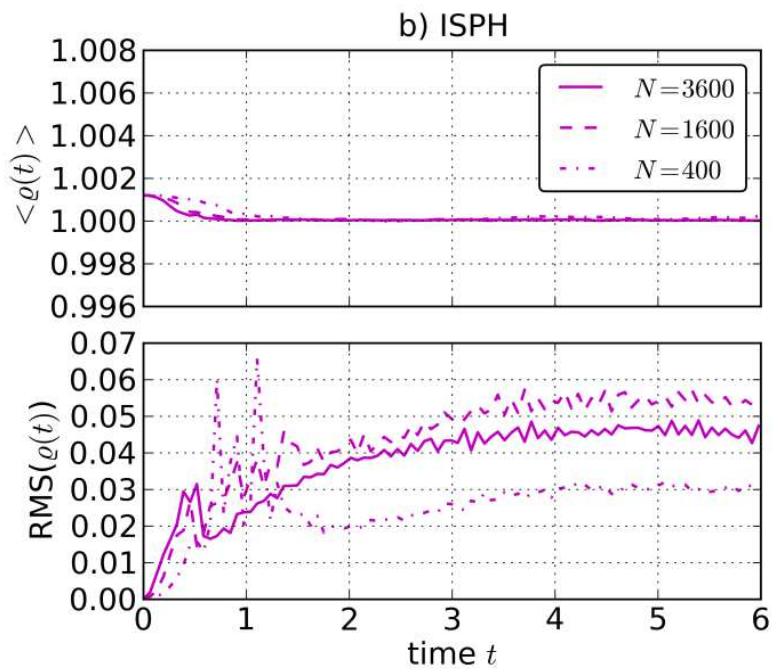

Figure 10. The density mean value and RMS coefficient obtained for the lid-driven cavity flow (Re=1000) performing: a) WCSPH and b) ISPH technique. Parameter $N$ denotes the number of particles in the domain.

particles, the ISPH profiles are much closer to the reference results. The simulations of the Couette flow uncover the numerical cost of the Poisson solver. Although the CPU time necessary to compute the Poisson equation is very high, the ISPH technique may perform with much larger timesteps (as in the case of the lid-driven cavity) and after all the efficiency of the ISPH is much greater than the WCSPH. Of course, increasing length of the time step, the error of results increases too. Due to this effect, the lid-driven cavity results, obtained via the ISPH, are characterized by greater RMS of the density than in the case of the WCSPH.

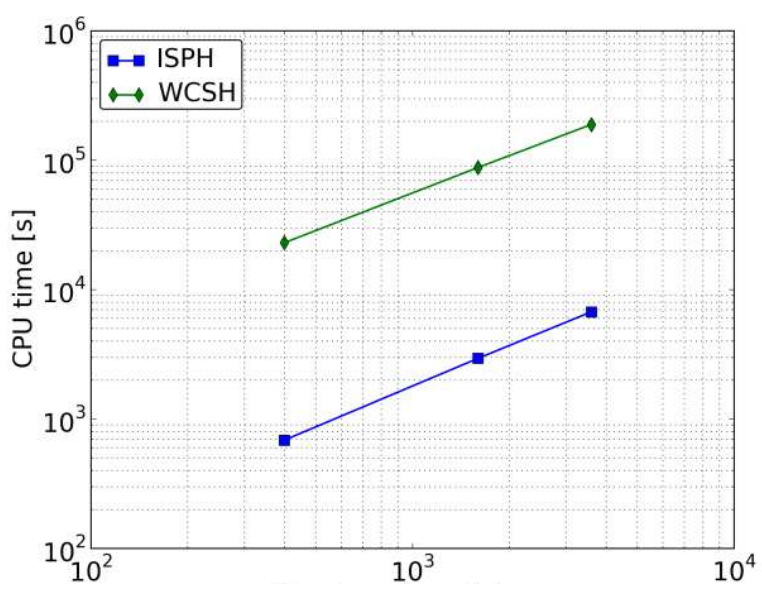

Figure 11. The CPU times to obtain the steady-state $(\mathrm{t}=55.0)$ solution of the lid-driven cavity $(R e=1000)$. 
correction term to a velocity field (4.5), they suggest the second correction to a density. This approach still needs a further research.

\section{References}

[1] J. J. Monaghan, "Smoothed Particles Hydrodynamics", Annu. Rev. Astron. Astrophy. 30, 542-547, 1992.

[2] S. J. Cummins, M. J. Rudman, “An SPH Projection Method", J. Comput. Phys. 152, 584-607, 1999.

[3] A. Valizadeh, M. Shafieefar, J. J. Monaghan, S. a. A. Selahi Neyshaboori, "Modeling Two-Phase Flows Using SPH Method", Journal of Applied Sciences 8 (21): 3817-3826, 2008.

[4] S. J. Cummins, M. J. Rudman, J. J. Monaghan, "Projection Methods and SPH", Monash University Applied Mechanics Reports and Preprints, 1997.

[5] P. W. Cleary, J. J. Monaghan, "Conduction Modelling Using Smoothed Particle Hydrodynamics", J. Comput. Phys. 148, 227-264, 1999.

[6] G. K. Batchelor, "An Introduction to Fluid Dynamics", Cambridge Univ. Press, 1967.

[7] D. J. Gryffits, "Introduction to electrodynamics 3rd ed.", Prentice Hall, 1999.

[8] J. P. Morris, "An Overview of the Smoothed Particle Hydrodynamics”, 1995.

[9] J. P. Morris, P. J. Fox, Y. Zhu, “Modeling Low Reynolds Number Incompressible Flows Using SPH", J. Comput. Phys. 136, 214-226, 1997.

[10]J. J. Monaghan, “On the problem of penetration in particle methods", J. Comput. Phys. 82, 1.

[11]P. M. Campbell, "Some new algorithms for boundary value problems in smooth particle hydrodynamics", Technical Report NA-TR-88296, Mission Research Corporation, Albuquerque, 1989.
[12]S. Shao, E.Y.M. Lo, “Incompressible SPH method for simulating Newtonian and non-Newtonian flows with a free surface", Adv. Water. Resour. 26, 787-800, 2003.

[13]U. Ghia, K.N. Ghia, C.T. Shin, "High Re-solutions for incompressible flow using the Navier-Stokes equations and a multigrid method", J. Comput. Phys. 48, 387-411, 1982.

[14]A. Wawreńczuk, "Modelling and computations of the flows with interface using Smoothed Particle Hydrodynamics (SPH)", PhD thesis, Institute of Fluid-Flow Machinery, Polish Academy of Sciences, Gdańsk, 2003.

[15]J. Pozorski, S. Wawreńczuk, "SPH computation of incompressible viscous flows", J. Theor. Appl. Mech. 40, 917-937, 2002 